\author{
BARANETSKij YA.O. ${ }^{1}$, KAlENYUK P.I. ${ }^{1}$, KOPACH M.I. ${ }^{2}$, SOlOMKO A.V. ${ }^{2}$
}

\title{
THE NONLOCAL BOUNDARY VALUE PROBLEM WITH PERTURBATIONS OF MIXED BOUNDARY CONDITIONS FOR AN ELLIPTIC EQUATION WITH CONSTANT COEFFICIENTS. I
}

\begin{abstract}
In this article we investigate a problem with nonlocal boundary conditions which are multipoint perturbations of mixed boundary conditions in the unit square $G$ using the Fourier method.

The properties of a generalized transformation operator $R: L_{2}(G) \rightarrow L_{2}(G)$ that reflects normalized eigenfunctions of the operator $L_{0}$ of the problem with mixed boundary conditions in the eigenfunctions of the operator $L$ for nonlocal problem with perturbations, are studied. We construct a system $V(L)$ of eigenfunctions of operator $L$. Also, we define conditions under which the system $V(L)$ is total and minimal in the space $L_{2}(G)$, and conditions under which it is a Riesz basis in the space $L_{2}(G)$. In the case if $V(L)$ is a Riesz basis in $L_{2}(G)$, we obtain sufficient conditions under which nonlocal problem has a unique solution in form of Fourier series by system $V(L)$.

Key words and phrases: differential equation with partial derivatives, eigenfunctions, Riesz basis.
\end{abstract}

\footnotetext{
${ }^{1}$ Lviv Polytechnic National University, 12 Bandera str., 79013, Lviv, Ukraine

${ }^{2}$ Vasyl Stefanyk Precarpathian National University, 57 Shevchenka str., 76018, Ivano-Frankivsk, Ukraine

E-mail: baryaromeukr.net (Baranetskij Ya.O.), kalenyuk@lp.edu.ua (Kalenyuk P.I.), kopachm2009@gmail.com (Kopach M.I.), ansolvas@gmail.com (Solomko A.V.)
}

\section{INTRODUCTION}

The fundamentals of the theory of linear differential equations in partial derivatives with constant coefficients were established by L. Ehrenpreis, L. Hermander, V. Malgrange, I. Petrovsky.

Boundary value problems in bounded domains for certain classes of differential equations with constant coefficients have been studied in [1-13]. This paper is a continuation of the investigations that were begun in [3-6].

For our investigation we will use the following notations. Let $G:=\left\{x:=\left(x_{1}, x_{2}\right) \in \mathbb{R}^{2}\right.$ : $\left.0<x_{1}, x_{2}<1\right\}, D_{1}, D_{2}$ are the operators of differentiation by the variables $x_{1}, x_{2}$ respectively; $H_{0}:=L_{2}(0,1), H_{1}:=L_{2}(G) ; H_{2}:=W_{2}^{2 n}(G)$ be a Sobolev space with a scalar product and norm respectively

$$
\begin{gathered}
\left(u, v ; H_{2}\right):=\left(u, v ; H_{1}\right)+\left(D_{1}^{2 n} u, D_{1}^{2 n} v ; H_{1}\right)+\left(D_{2}^{2 n} u, D_{2}^{2 n} v ; H_{1}\right),\left\|u ; H_{2}\right\|:=\sqrt{\left(u, u ; H_{2}\right)} \\
W:=\left\{v \in C[0,1]: v^{(s)} \in C[0,1], s=1, \ldots, 2 n-1, v^{(2 n)} \in H_{0}\right\} \\
H_{0, s}:=\left\{u(t) \in H_{0}: u(t) \equiv(-1)^{s} u(1-t)\right\}, s \in\{0,1\}
\end{gathered}
$$

$\mathrm{y} \Delta \mathrm{K} 517.927 .5$

2010 Mathematics Subject Classification:34G10, 34K10, 34K30, 34L10. 
$W_{r}:=W \cap H_{0, r}, r=0,1$; and $\left[H_{0}\right]$ be a set of linear continuous operators on the space $H_{0}$. Let us consider the boundary value problem

$$
\begin{gathered}
L\left(-D_{1}^{2},-D_{2}^{2}\right) u:=\sum_{j=0}^{n} a_{j} D_{1}^{2 j} D_{2}^{2 n-2 j} u=f(x), x \in G, \\
\ell_{s, 1} u:=\left.D_{1}^{2 s-2} u\right|_{x_{1}=0}+\left.D_{1}^{2 s-2} u\right|_{x_{1}=1}+\ell_{s, 1}^{0} u=0, \\
\ell_{n+s, 1} u:=\left.D_{1}^{2 s-2} u\right|_{x_{1}=0}-\left.D_{1}^{2 s-2} u\right|_{x_{1}=1}=0, \\
\ell_{s, 2} u:=\left.D_{2}^{2 s-2} u\right|_{x_{1}=0}+\left.D_{2}^{2 s-2} u\right|_{x_{1}=1}=0, \\
\ell_{n+s, 2} u:=\left.D_{2}^{2 s-1} u\right|_{x_{2}=0}+\left.D_{2}^{2 s-1} u\right|_{x_{2}=1}=0, s=1, \ldots, n,
\end{gathered}
$$

where

$$
\begin{gathered}
\ell_{s, 1}^{0} u:=\left.\sum_{q=0}^{k_{s, 1}} \sum_{r=0}^{n_{1}} b_{s, q, r} D_{1}^{q} u\right|_{x_{1}=x_{1, r}}, s=1, \ldots, n, \\
0=x_{1,1}<x_{1,2}<\cdots<x_{1, n_{1}} \leq 1, a_{j}, b_{s, q, r} \in \mathbb{R}, \\
q=0,1, \ldots, k_{s, 1}, k_{s, 1}<2 n, r=0,1, \ldots, n_{1}, s=1, \ldots, n, j=0,1, \ldots, n .
\end{gathered}
$$

Let $L: H_{1} \rightarrow H_{1}$ be the operator of the problem (1)-(6) and

$$
\begin{gathered}
L u:=L\left(-D_{1}^{2},-D_{2}^{2}\right) u, u \in D(L), \\
D(L):=\left\{u \in H_{2}: \ell_{s, j} u=0, s=1, \ldots, 2 n, j=1,2\right\} .
\end{gathered}
$$

Definition. The function $y \in D(L)$, that satisfies equality $\left\|L\left(-D_{1}^{2},-D_{2}^{2}\right) y-f ; H_{1}\right\|=0$, is called a solution of problem (1)-(6).

Let us consider the following assumptions and theorems, that are necessary for further investigation.

1. Assumption $P_{1}: b_{s, q, r}=-(-1)^{q} b_{s, q, n_{1}-r}, x_{1, r}=1-x_{1, n_{1}-r}, r=0,1, \ldots, n_{1}, s=1, \ldots, n$.

2. Assumption $P_{2}: k_{s, 1} \leq 2 s-2, s=1, \ldots, n$.

3. Assumption $P_{3}$ : for any real numbers $\mu_{1}, \mu_{2}$ the positive number $C_{1}(L)$ exists, that the inequality $C_{1}(L)|\mu|^{2 n} \leq\left|L\left(\mu_{1}, \mu_{2}\right)\right|, \mu:=\left(\mu_{1}, \mu_{2}\right),|\mu|^{2}:=\left|\mu_{1}\right|^{2}+\left|\mu_{2}\right|^{2}$, holds.

Theorem 1. Let Assumption $P_{1}$ holds. Then, for an arbitrary $a_{q} \in \mathbb{R}, q=0,1, \ldots, n, b_{s, q, r} \in \mathbb{R}$, the operator $L$ has a set of eigenvalues

$$
\sigma:=\left\{\lambda_{k, m}:=L\left(\mu_{1, k}, \mu_{2, m}\right), \mu_{1, k}=\pi^{2} k^{2}, \mu_{2, m}=\pi^{2}(2 m-1)^{2}, k \in \mathbb{N}, m \in \mathbb{N}\right\},
$$

and the system $V(L)$ of eigenfunctions, which is complete and minimal in the space $H_{1}$.

Theorem 2. Let Assumptions $P_{1}-P_{3}$ hold. Then, the operator $L$ has the system $V(L)$ of eigenfunctions, which is the Riesz basis of the space $H_{1}$.

Theorem 3. Let Assumptions $P_{1}-P_{3}$ hold. Then, for arbitrary function $f \in H_{1}$ the unique solution of problem (1)-(6) exists. 
Let $A_{0}$ be the operator of boundary problem in the space $H_{0}$ :

$$
\begin{gathered}
-z^{(2)}(t)=g(t), t \in(0,1), z(0)=z(1)=0 ; \\
A_{0} z:=-z^{(2)}(t), z(t) \in D\left(A_{0}\right), D\left(A_{0}\right):=\left\{z \in W_{2}^{2}(0,1): z(0)=z(1)=0\right\} ; \\
T_{1}:=\left\{\tau_{1, s, k}(t) \in H_{0}: \tau_{1, s, k}(t):=\sqrt{2} \sin \rho_{s, k} t, \rho_{s, k}=\pi(2 k+s-1), k \in \mathbb{N}, s=0,1\right\} ; \\
T_{1, s}:=\left\{\tau_{1, s, k}(t) \in H_{0, s}, k \in \mathbb{N}\right\}, s=0,1 ; \\
\sigma\left(A_{0}\right):=\left\{\mu_{1, k}=\pi^{2} k^{2}, k \in \mathbb{N}\right\} .
\end{gathered}
$$

Lemma 1. The operator $A_{0}$ has the point spectrum $\sigma\left(A_{0}\right)$ and system of eigenfunctions $T_{1}$.

Proof. A direct substitution proves that the elements of system $T_{1}$ are the eigenfunctions of operator $A_{0}$, which correspond to the eigenvalues $\sigma\left(A_{0}\right)$.

Taking into account that the subsystem of eigenfunctions $T_{1, s}$ of the operator $A_{0}$ is an orthonormal basis of spaces $H_{0, s}, s=0,1$, we obtain the statement of the lemma.

Let $\Theta=\left\{\theta_{k}\right\}_{k=1}^{\infty}$ be any sequence of real numbers. We consider the operator $A_{\Theta}: H_{0} \rightarrow$ $H_{0}$, which has a set of eigenvalues $\sigma\left(A_{0}\right)$, and the system of eigenfunctions

$$
\begin{aligned}
& V\left(A_{\Theta}\right):=\left\{v_{s, k}\left(t, A_{\Theta}\right) \in H_{0}: v_{0, k}\left(t, A_{\Theta}\right):=\tau_{1,0, k}(t),\right. \\
&\left.v_{1, k}\left(t, A_{\Theta}\right):=\tau_{1,1, k}(t)+\theta_{k} \sqrt{2} \cos 2 k \pi t, k \in \mathbb{N}\right\} .
\end{aligned}
$$

Lemma 2. For an arbitrary sequence $\Theta$ the system of functions $V\left(A_{\Theta}\right)$ is complete and minimal in the space $H_{0}$. The system of functions $V\left(A_{\Theta}\right)$ is the Riesz basis of this space if and only if the sequence $\Theta$ is bounded.

Proof. Suppose that the system $V\left(A_{\Theta}\right)$ is not complete in the space $H_{0}$.

Let us suppose that there exist functions $f=f_{0}+f_{1} \in H_{0}$, and $f_{s} \in H_{0, s}, s=0,1$, for which the conditions of orthogonality hold:

$$
\left(f, v_{s, k}\left(t, A_{\Theta}\right) ; H_{0}\right)=0, s=0,1, k \in \mathbb{N} .
$$

Taking into account, that the system of functions $\tau_{1,0, q}(t)=v_{0, q}\left(t, A_{\Theta}\right), q \in \mathbb{N}$, is an orthonormal basis of the space $H_{0,0}$ with respect to the condition of orthogonality, we obtain $f_{0}=0$. Thus $f=f_{1} \in H_{0,1}$.

According to the condition of orthogonality we have the relation

$$
\left(f, v_{1, k}\left(t, A_{\Theta}\right) ; H_{0}\right)=\left(f, \tau_{1,1, k}(t) ; H_{0}\right)=0, k \in \mathbb{N} \text {. }
$$

Taking into account the totality of the system of functions $V_{1}\left(L_{0}\right)=T_{1,1}$ in the space $H_{0,1}$, we have $f=f_{1} \equiv 0$. Thus the system $V\left(A_{\Theta}\right)$ is total (complete) in the space $H_{0}$. Therefore, the operator $A_{\Theta}$ is defined on a dense set of the space $H_{0}$.

In the space $H_{0}$ let us define the operators

$$
R\left(A_{\Theta}\right):=E+S\left(A_{\Theta}\right), \quad S\left(A_{\Theta}\right) \tau_{1,0, q}(t):=0, S\left(A_{\Theta}\right) \tau_{1,1, q}(t):=\theta_{q} \sqrt{2} \cos 2 q \pi t \in H_{0,0}, q \in \mathbb{N} .
$$

According to equality $S^{2}\left(A_{\Theta}\right)=0$ we get the relation $R^{-1}\left(A_{\Theta}\right)=E-S\left(A_{\Theta}\right)$. Therefore, the system of functions $V\left(A_{\Theta}\right)$ is minimal in the space $H_{0}$. Let us prove the second part of the lemma. 
Necessity. We choose any bounded sequence $\Theta$ and show that $S\left(A_{\Theta}\right): H_{0} \rightarrow H_{0}$ is a bounded operator.

Let us expand an arbitrary function $h \in H_{0}$ into Fourier series

$$
h=\sum_{k=1}^{\infty} \sum_{j=0}^{1} h_{j, k} \tau_{1, j, k}(t) .
$$

Consider $S\left(A_{\Theta}\right) h=\sum_{k=1}^{\infty} \theta_{m} h_{1, k} \sqrt{2} \cos 2 k \pi t$.

Taking into account that the system of functions $\{1, \cos 2 k \pi t, k \in \mathbb{N}\}$ is an orthonormal basis of $H_{0,0}$ and using Cauchy's inequality, we obtain

$$
\left\|S\left(A_{\Theta}\right) h ; H_{0}\right\|^{2} \leq C_{1}\left\|h ; H_{0}\right\|^{2}, C_{1}=\max \left|\theta_{k}\right|^{2} .
$$

Thus $S\left(A_{\Theta}\right) \in\left[H_{0}\right]$.

Taking into account the relation $R^{-1}\left(A_{\Theta}\right)=E-S\left(A_{\Theta}\right)$, we obtain an estimate

$$
\left\|R^{-1}\left(A_{\Theta}\right) ;\left[H_{0}\right]\right\|^{2} \leq C_{2}, C_{2}=2+2 C_{1} .
$$

Thus the system $V\left(A_{\Theta}\right)$ is the Riesz basis by definition.

Sufficiency. Let $V\left(A_{\Theta}\right)$ be the Riesz basis in the space $H_{0}$. Therefore, this system is almost normalized. Thus, for any positive numbers $C_{3} \leq C_{4}$ the next inequality holds:

$$
C_{3} \leq\left\|v_{s, m}\left(t, A_{\Theta}\right) ; H_{0}\right\| \leq C_{4}<\infty, m \in \mathbb{N} .
$$

Taking into account the equalities

$$
\left\|v_{0, k}\left(t, A_{\Theta}\right) ; H_{0}\right\|=1,\left\|v_{1, m}\left(t, A_{\Theta}\right) ; H_{0}\right\|=1+\left|\theta_{m}\right|, k=0,1, \ldots, m \in \mathbb{N},
$$

we obtain the proof of sufficiency.

Let $B_{0}$ be the operator of spectral problem

$$
\begin{gathered}
-z^{(2)}(t)=\mu z(t), \mu \in \mathbb{C}, \\
\ell_{1} z:=z(0)+z(1)=0, \\
\ell_{2} z:=z^{(1)}(0)+z^{(1)}(1)=0, \\
B_{0} z:=-z^{(2)}(t), z(t) \in D\left(B_{0}\right), D\left(B_{0}\right):=\left\{z \in W_{2}^{2}(0,1): \ell_{s} z=0, s=1,2\right\}, \\
T_{2}:=\left\{\tau_{2, r, m}(t) \in H_{0}: \tau_{2,0, m}(t):=\sqrt{2} \sin \pi(2 m-1) t, \tau_{2,1, m}(t):=\sqrt{2} \cos \pi(2 m-1) t, m \in \mathbb{N}\right\}, \\
\sigma\left(B_{0}\right):=\left\{\mu_{2, m}=\pi^{2}(2 m-1)^{2}, m \in \mathbb{N}\right\} .
\end{gathered}
$$

Lemma 3. The operator $B_{0}$ has the point spectrum $\sigma\left(B_{0}\right)$ and system of eigenfunctions $T_{2}$.

Proof. After performing a direct substitution we obtain that

$$
\tau_{2, r, m}(t) \in D\left(B_{0}\right), \quad-\tau_{2, r, m}^{(2)}(t)=\mu_{2, m} \tau_{2, r, m}(t), \quad r=0,1, \quad m \in \mathbb{N} .
$$

Thus operator $L_{0}$ has the system of eigenfunctions $V\left(L_{0}\right)$, which corresponds to the set of eigenvalues $\sigma$. 
For the equation (1) we consider the boundary conditions $\ell_{0, s, j} u=0, s=1, \ldots, 2 n, j=1,2$, which are the partial case of boundary conditions (2)-(6) for $\ell_{s, 1}^{1} u=0, s=1, \ldots, n$.

Let $L_{0}: H_{1} \rightarrow H_{1}$ be the operator of the obtained problem

$$
L_{0} u:=L\left(-D_{1}^{2},-D_{2}^{2}\right) u, u \in D\left(L_{0}\right), D\left(L_{0}\right):=\left\{u \in H_{2}: \ell_{0, s, j} u=0, s=1, \ldots, 2 n, j=1,2\right\}
$$

and

$$
V\left(L_{0}\right):=\left\{v_{r, s, k, m}\left(x, L_{0}\right) \in H_{1}: v_{r, s, k, m}\left(x, L_{0}\right):=\tau_{1, s, k}\left(x_{1}\right) \tau_{2, r, m}\left(x_{2}\right), r, s \in\{0,1\}, m, k \in \mathbb{N}\right\}
$$

be the orthonormal basis of the space $H_{1}$.

Considering the ratio $L_{0}=(-1)^{n} \sum_{s=0}^{n} A_{0}^{s} B_{0}^{n-s}$, we obtain the following statement.

Lemma 4. The operator $L_{0}$ has eigenvalues (7) and the system of eigenfunctions $V\left(L_{0}\right)$.

\section{THE NON SELF-AJOINT PROBLEM FOR A DIFFERENTIAL EQUATION OF EVEN ORDER}

For any fixed $p \in\{1, \ldots, n\}$ we consider the problem

$$
\begin{gathered}
L\left(-D_{1}^{2},-D_{2}^{2}\right) u:=\sum_{s=0}^{n} a_{s} D_{1}^{2 s} D_{2}^{2 n-2 s} u(x)=\lambda u(x), x \in G, \lambda \in \mathbb{C}, \\
\ell_{1, s, 1} u:=\left.D_{1}^{2 s-2} u\right|_{x_{1}=0}+\left.D_{1}^{2 s-2} u\right|_{x_{1}=1}=0, s \neq p, s=1, \ldots, n, \\
\ell_{1, p, 1} u:=\left.D_{1}^{2 p-2} u\right|_{x_{1}=0}+\left.D_{1}^{2 p-2} u\right|_{x_{1}=1}+\ell_{p, 1}^{0} u=0, \\
\ell_{1, n+s, 1} u:=\left.D_{1}^{2 s-2} u\right|_{x_{1}=0}-\left.D_{1}^{2 s-2} u\right|_{x_{1}=1}=0, s \neq p, s=1, \ldots, n, \\
\ell_{1, s, 2} u:=\left.D_{2}^{2 s-2} u\right|_{x_{2}=0}+\left.D_{2}^{2 s-2} u\right|_{x_{2}=1}=0, s=1, \ldots, n, \\
\ell_{1, n+s, 2} u:=\left.D_{2}^{2 s-1} u\right|_{x_{2}=0}+\left.D_{2}^{2 s-1} u\right|_{x_{2}=1}=0, s=1, \ldots, n .
\end{gathered}
$$

Let $L_{1, p}$ be the operator of the problem (8)-(13):

$$
\begin{aligned}
L_{1, p} u & :=L\left(-D_{1}^{2},-D_{2}^{2}\right) u, u \in D\left(L_{1, p}\right), \\
D\left(L_{1, p}\right) & :=\left\{u \in H_{2}: \ell_{1, r, j} u=0, r=1, \ldots, 2 n, j=1,2\right\},
\end{aligned}
$$

and $V\left(L_{1, p}\right)$ be the system of eigenfunctions of the operator $L_{1, p}$.

For any fixed $m \in \mathbb{N}$ let's consider the solutions of problem (8)-(13) in the form of product

$$
u(x):=z\left(x_{1}\right) \tau_{2, s, m}\left(x_{2}\right), s \in\{0,1\} .
$$

To determine the unknown function $z\left(x_{1}\right)$, we obtain the problem for eigenvalues

$$
\begin{gathered}
\sum_{q=0}^{n} a_{q}(-1)^{n-s} \mu_{2, m}^{n-q} z^{(2 q)}\left(x_{1}\right)=\lambda z\left(x_{1}\right), \quad x_{1} \in(0,1), \lambda \in \mathbb{C}, \\
l_{s, 1}^{1} z:=z^{(2 s-2)}(0)+z^{(2 s-2)}(1)=0, s \neq p, s=1, \ldots, n, \\
l_{p, 1}^{1} z:=z^{(2 p-2)}(0)+z^{(2 p-2)}(1)+l_{p, 1}^{0} z=0,
\end{gathered}
$$




$$
l_{n+s, 1}^{1} z:=z^{(2 s-2)}(0)-z^{(2 s-2)}(1)=0, s=1, \ldots, n,
$$

where

$$
l_{p, 1}^{0} z:=\sum_{q=0}^{k_{p, 1}} \sum_{r=0}^{n_{1}} b_{p, q, r} z^{(q)}\left(x_{1, r}\right), p=1, \ldots, n .
$$

Let $L_{1, p, m}$ be the operator of problem (14)-(18):

$$
\begin{aligned}
L_{1, p, m} z & :=\sum_{s=0}^{n} a_{s}(-1)^{m-s} \mu_{2, m}^{n-s} z^{(2 s)}, \quad z \in D\left(L_{1, p, m}\right), \\
D\left(L_{1, p, m}\right) & :=\left\{z \in W: l_{j, 1}^{1} z=0, j=1, \ldots, 2 n\right\} .
\end{aligned}
$$

Lemma 5. Let Assumption $P_{1}$ holds. Therefore, for any $a_{q} \in \mathbb{R}, b_{p, q, r} \in \mathbb{R}, q=0,1, \ldots, k_{p, 1}$, $r=0,1, \ldots, n_{1}, m, p \in \mathbb{N}$, the operator $L_{1, p, m}$ has the set of eigenvalues $\sigma_{m}:=\left\{\lambda_{k, m} \in \sigma\right.$, $k \in \mathbb{N}\}$, and the system of eigenfunctions $V\left(L_{1, p, m}\right)$, which is complete and minimal in the space $H_{0}$.

Proof. The solutions $\omega_{r, m}(\lambda), r=1, \ldots, n$, of equation $\sum_{s=0}^{n} a_{s}(-1)^{n-s} \mu_{2, m}^{n-s} \omega^{2 s}=\lambda$, which is characteristic for equations (14), we choose to fulfill the conditions

$$
\operatorname{Re} \omega_{n, m}(\lambda) \leq \operatorname{Re} \omega_{n-1, m}(\lambda) \leq \cdots \leq \operatorname{Re} \omega_{1, m}(\lambda) \leq 0 .
$$

Let us determine the functions

$$
\begin{gathered}
z_{q, m}\left(x_{1}, \lambda\right):=\frac{1}{2}\left(\exp \omega_{q, m}(\lambda) x_{1}+\exp \omega_{q, m}(\lambda)\left(1-x_{1}\right)\right) \in H_{0,0}, q=1, \ldots, n, \\
z_{n+q, m}\left(x_{1}, \lambda\right):=\frac{1}{2}\left(\exp \omega_{q, m}(\lambda) x_{1}-\exp \omega_{q, m}(\lambda)\left(1-x_{1}\right)\right) \in H_{0,1}, q=1, \ldots, n, \\
z_{m}\left(x_{1}\right)=\sum_{j=1}^{2 n} c_{j} z_{j, m}\left(x_{1}, \lambda\right), c_{j} \in \mathbb{R} .
\end{gathered}
$$

Substituting expression (19) into boundary conditions (15)-(17), we obtain an equation for determining of eigenvalues for operator $L_{1, p, m}$ :

$$
\Delta_{m}(\lambda)=\operatorname{det}\left(l_{q, 1}^{1} z_{j, m}(x, \lambda)\right)_{j, q=1}^{2 n}=0 .
$$

According to the relations $z_{r n+q, m}\left(x_{1}, \lambda\right) \in H_{0, r}, l_{q+s n}^{1} \in W_{s}^{*}, s, r \in\{0,1\}, l_{p, 1}^{0} \in W_{1}^{*}$, we obtain

$$
\begin{gathered}
l_{n+q, 1}^{1} z_{j, m}\left(x_{1}, \lambda\right)=0, l_{q, 1}^{1} z_{n+j, m}\left(x_{1}, \lambda\right)=0, j, q=1, \ldots, n, \\
\Delta_{m}(\lambda)=\Delta_{0, m}(\lambda) \Delta_{1, m}(\lambda), \\
\Delta_{m}(\lambda)=\prod_{q=1}^{n}\left(1-e^{2 \omega_{q, m}(\lambda)}\right) \prod_{1 \leq j<q \leq n}\left(\omega_{j, m}(\lambda)-\omega_{q, m}(\lambda)\right)^{2}=0 .
\end{gathered}
$$

Let $\omega_{r, k, m}$ be roots of the equation (20) for $\lambda=\lambda_{k, m}$, which are selected so that $\omega_{1, k, m}=\imath \pi k, \quad \operatorname{Re} \omega_{n, k, m} \leq \operatorname{Re} \omega_{n-1, k, m} \leq \cdots \leq \operatorname{Re} \omega_{1, k, m} \leq 0, k \in \mathbb{N}$. Substituting expression (19) in boundary conditions (15)-(17), we can find the eigenfunctions of the operator $L_{1, p, m}$ :

$$
v_{0, k}\left(x_{1}, L_{1, p, m}\right)=\sqrt{2} \sin \rho_{0, k} x_{1}, \rho_{0, k}=\pi(2 k-1), k \in \mathbb{N} .
$$


Let us define the system of functions

$$
\begin{gathered}
z_{1,1, k, m}\left(x_{1}\right)=\sqrt{2} \cos \rho_{1, k} x_{1}, \rho_{1, k}=2 k \pi, k \in \mathbb{N}, \\
z_{1, q, k, m}\left(x_{1}\right):=\frac{1}{2}\left(1+\exp \omega_{q, k, m}\right)^{-1}\left(\exp \omega_{q, k, m} x_{1}+\exp \omega_{q, k, m}\left(1-x_{1}\right)\right), k \in \mathbb{N},
\end{gathered}
$$

and a square matrix of order $n$, elements of which we define by the following rule: $p$ th row is defined by functions (22), (23), and elements of other rows is defined by numbers

$$
\begin{gathered}
\vartheta_{q, r, k, m}:=\rho_{1, k}^{2-2 r} l_{1, r, 1} z_{1, q, k, m}, v_{q, r, k, m}=\rho_{1, k}^{2-2 r} \omega_{q, k, m}^{2 r-2}, q=2,3, \ldots, n, r \neq p, r=1, \ldots, n . \\
\vartheta_{1, r, k, m}=2 \sqrt{2}, r \neq p,=2,3, \ldots, n, k \in \mathbb{N} .
\end{gathered}
$$

Determinant of the given matrix is denoted by $z_{2, p, k, m}\left(x_{1}\right), k \in \mathbb{N}$.

Remark 1. For any fixed $m \in \mathbb{N}$ and $k \rightarrow \infty$, we obtain the relation

$$
\begin{gathered}
\delta_{1, k, m}:=\omega_{1, k, m} \rho_{1, k}^{-1}=\imath, \\
\delta_{q, k, m}:=\rho_{1, k}^{-1} \omega_{q, k, m}=\varepsilon_{q}\left(1+O\left(k^{-1}\right)\right),
\end{gathered}
$$

where $\varepsilon_{q}$ are the solutions of equation $(-1)^{n}(\varepsilon)^{2 n}=1, \varepsilon_{1}=1, \operatorname{Im} \varepsilon_{q}<0, q=2,3, \ldots, n$.

Substituting function $z_{2, p, k, m}\left(x_{1}\right)$ in boundary conditions (14)-(17), we obtain the equalities

$$
\ell_{1, s, 1} z_{2, p, k, m}=0, s \neq p, l_{1, p, 1} z_{2, p, k, m}:=c_{p, k, m}, s=1, \ldots, 2 n, k \in \mathbb{N},
$$

where $c_{p, k, m}=\sqrt{2} 2 \rho_{1, k}^{2 p-2} W_{k, m}, W_{k, m}=W\left(\delta_{1, k, m}^{2} \ldots, \delta_{n, k, m}^{2}\right)$ is Vandermonde determinant of order $n$, which is constructed by numbers $\delta_{q, k, m}^{2}, q=1, \ldots, n$.

Remark 2. For arbitrary $m \in \mathbb{N}$ and $k \rightarrow \infty$ the number sequence $\left\{W_{k, m}\right\}_{k=1}^{\infty}$ converges to Vandermonde determinant $W\left(\varepsilon_{1}^{2}, \varepsilon_{2}^{2}, \ldots, \varepsilon_{n}^{2}\right)$, which is constructed by numbers $\varepsilon_{1}^{2}, \ldots, \varepsilon_{n}^{2}$.

Therefore, $\vartheta_{q, r, k, m}=\varepsilon_{q}^{2 r-2}\left(1+O\left(\frac{1}{k}\right)\right), k \rightarrow \infty, q=1, \ldots, n$.

Thus, the positive numbers $C_{5}, C_{6}$ exist such that the following inequality holds:

$$
0<C_{5} \leq\left|c_{p, k, m}\right| \rho_{1, k}^{2-2 p} \leq C_{6}<\infty, k \in \mathbb{N} \text {. }
$$

Let us choose the functions

$$
z_{3, p, k, m}\left(x_{1}\right):=W_{k, m}^{-1} z_{2, p, k, m}\left(x_{1}\right), k \in \mathbb{N} .
$$

Taking into account equalities (24), we obtain the relations

$$
\ell_{1, s}^{1} z_{3, p, k, m}=0, s \neq p, \ell_{1, p}^{1} z_{3, p, k, m}\left(x_{1}\right)=2 \sqrt{2} \rho_{1, k}^{2 p-2}, s=1, \ldots, n .
$$

Let $\Delta_{j, s, k, m}:=\operatorname{det}\left(\vartheta_{q, r, k, m}\right)_{q, r=1, n}^{q \neq j, r \neq s}$. Consider the functions $y_{p, k, m}\left(x_{1}\right):=\Delta_{1,1, k, m}^{-1} z_{3, p, k, m}\left(x_{1}\right)$,

$$
y_{p, k, m}\left(x_{1}\right)=z_{1,1, k, m}\left(x_{1}\right)+\sum_{j=2}^{n} \gamma_{j, p, k, m} z_{1, j, k, m}\left(x_{1}\right), k \in \mathbb{N},
$$

where $\gamma_{j, p, k, m}=\Delta_{1, p, k, m}^{-1} \Delta_{j, p, k, m}, j=2,3, \ldots, n$. 
From formulas (24)-(26) we obtain

$$
y_{p, k, m}\left(x_{1}\right)=c_{1, p, k, m} z_{2, p, k, m}\left(x_{1}\right)
$$

where

$$
c_{1, p, k, m}=W_{k, m}^{-1} \Delta_{1, p, k, m}, C_{7}<c_{1, p, k, m}<C_{8}<\infty
$$

Therefore,

$$
l_{1, p}^{1} y_{p, k, m}\left(x_{1}\right)=c_{1, p, k, m} 2 \sqrt{2} \rho_{1, k}^{2 p-2}, l_{1, s}^{1} y_{p, k, m}\left(x_{1}\right)=0, s \neq p, s=1, \ldots, n .
$$

The eigenfunctions $v_{1, k}\left(x_{1}, L_{1, p, m}\right)$ of the operator $L_{1, p, m}$ we define by the equality

$$
v_{1, k}\left(x_{1}, L_{1, p, m}\right):=\tau_{1,1, k}\left(x_{1}\right)+\eta_{p, k, m} y_{p, k, m}\left(x_{1}\right), k \in \mathbb{N}
$$

To determine the unknown parameters $\eta_{p, k, m}$, we substitute the expression (27) in the boundary conditions (16), (17).

Taking into account (24), we obtain

$$
\eta_{p, k, m}=(-1)^{p} \sqrt{8^{-1}} c_{1, p, k, m}^{-1} \rho_{1, k}^{2-2 p} l_{1, p}^{1} \tau_{1,1, k}, k \in \mathbb{N} .
$$

Thus, the operator $L_{1, p, m}$ has the system $V\left(L_{1, p, m}\right)$ of eigenfunctions (21), (24), (28).

The completeness of the system of functions $V\left(L_{1, p, m}\right)$ in the space $H_{0}$ is proved from the opposite, like in the proof of the Lemma 2.

Let us consider the operators

$$
\begin{gathered}
R\left(L_{1, p, m}\right), S\left(L_{1, p, m}\right): H_{0} \rightarrow H_{0}, \quad R\left(L_{1, p, m}\right)=E+S\left(L_{1, p, m}\right), \\
R\left(L_{1, p, m}\right) \tau_{1,0, k}\left(x_{1}\right):=\tau_{1,0, k}\left(x_{1}\right), \quad R\left(L_{1, p, m}\right) \tau_{1,1, k}\left(x_{1}\right):=v_{1, k}\left(x_{1}, L_{1, p, m}\right), k \in \mathbb{N} .
\end{gathered}
$$

From the definition of operator $S\left(L_{1, p, m}\right)$ we obtain $S\left(L_{1, p, m}\right): H_{0,0} \rightarrow 0, S\left(L_{1, p, m}\right): H_{0,1} \rightarrow$ $H_{0,0}, S^{2}\left(L_{1, p, m}\right)=0, R^{-1}\left(L_{1, p, m}\right)=E-S\left(L_{1, p, m}\right)$. Therefore, the system of functions $V\left(L_{1, p, m}\right)$ is minimal in the space $H_{0}$. Lemma 5 is proved.

Let $\theta_{k}=\eta_{p, k, m}$, then $A_{p, m}:=A_{\Theta}, k, m \in \mathbb{N}, p \in\{1, \ldots, n\}$.

Lemma 6. If $\left\{\eta_{p, k, m}\right\}_{k=1}^{\infty}$ is a bounded sequence, then the system of functions $V\left(L_{1, p, m}\right)$ is the Riesz basis in the space $H_{0}$.

Proof. Taking into account the definition of the function $y_{p, k, m}\left(x_{1}\right)$ and the choice of numbers $\omega_{q, k, m}, q=1, \ldots, n$, we can conclude: if $\theta_{k}=\eta_{p, k, m}, k \in \mathbb{N}, p \in\{1, \ldots, n\}$, is a bounded sequence, then the systems of functions $V\left(L_{1, p, m}\right), V\left(A_{p, m}\right)$ are quadratically approximate for every $m \in \mathbb{N}, p \in\{1, \ldots, n\}$.

Therefore, taking into account the Lemma 5 and the theorem N.K. Bari [10], we obtain the statement of Lemma 6.

Let us choose an arbitrary sequence of real numbers $\Theta=\left\{\theta_{k}\right\}_{k=1}^{\infty}$, and define the operator $A_{\Theta, p, m}: H_{0} \rightarrow H_{0}$, which has the set of eigenvalues $\sigma_{1, m}=\left\{\lambda_{k, m} \in \sigma, k \in \mathbb{N}\right\}$ and the system $V\left(A_{\Theta, p, m}\right):=\left\{v_{s, k, m}\left(x_{1}, A_{\Theta, p, m}\right) \in H_{0}: s=0,1, k \in \mathbb{N}\right\}$ of eigenfunctions

$$
v_{0, k, m}\left(x_{1}, A_{\Theta, p, m}\right):=\tau_{1,0, k}\left(x_{1}\right), v_{1, k, m}\left(x_{1}, A_{\Theta, p, m}\right):=\tau_{1,1, k}\left(x_{1}\right)+\theta_{k} y_{p, k, m}\left(x_{1}\right), k \in \mathbb{N}
$$


Consider the operators

$$
\begin{aligned}
& R\left(A_{\Theta, p, m}\right):=E+S\left(A_{\Theta, p, m}\right), \\
& S\left(A_{\Theta, p, m}\right) \tau_{1,0, k}\left(x_{1}\right):=0, \\
& S\left(A_{\Theta, p, m}\right) \tau_{1,1, k}\left(x_{1}\right):=\theta_{k} y_{p, k, m}\left(x_{1}\right), k \in \mathbb{N} .
\end{aligned}
$$

Let $\Gamma_{1, p}\left(L_{0, m}\right)$ be the set of operators, which have purely point spectrum $\sigma_{1, m}$ and the system of eigenfunctions (29).

We define on $\Gamma_{1, p}\left(L_{0, m}\right)$ the commutative multiplication operation

$$
\begin{gathered}
R\left(A_{\Theta_{1}, p, m}\right) R\left(A_{\Theta_{2}, p, m}\right)=E+S\left(A_{\Theta_{1}, p, m}\right)+S\left(A_{\Theta_{2}, p, m}\right)=R\left(A_{\Theta_{2}, p, m}\right) R\left(A_{\Theta_{1}, p, m}\right), \\
A_{\Theta_{2}, p, m}, A_{\Theta_{1}, p, m} \in \Gamma_{1, p}\left(L_{0}\right),
\end{gathered}
$$

and inverse operator $R^{-1}\left(A_{\Theta, p, m}\right)=E-S\left(A_{\Theta, p, m}\right), A_{\Theta, p, m} \in \Gamma_{1}\left(L_{0, m}\right)$.

Lemma 7. For any real numbers $\theta_{q} \in \mathbb{R}, q \in \mathbb{N}$, the system of functions $V\left(A_{\Theta, p, m}\right)$ is complete and minimal in the space $H_{0}$. The system of functions $V\left(A_{\Theta, p, m}\right)$ is the Riesz basis in $H_{0}$ if and only if the sequence $\Theta$ is bounded.

Proof. The lemma can be proved by the schema of proof the Lemma 2.

We define by the formulas

$$
v_{s, r, k, m}\left(x, L_{1, p}\right):=v_{s, k}\left(x_{1}, L_{1, p, m}\right) \tau_{2, r, m}\left(x_{2}\right), s, r \in\{0,1\}, k, m \in \mathbb{N},
$$

the eigenfunctions of operator $L_{1, p}$.

Lemma 8. Suppose that the Assumption $P_{1}$ holds. Then, for arbitrary $a_{s} \in \mathbb{R}, b_{p, q, r} \in \mathbb{R}$, the operator $L_{1, p}$ has the point spectrum $\sigma$, and the system of eigenfunctions $V\left(L_{1, p}\right):=$ $\left\{v_{s, r, k, m}\left(x, L_{1, p}\right), s, r \in\{0,1\}, k, m \in \mathbb{N}\right\}$, which is complete and minimal in $H_{1}$.

If the Assumptions $P_{1}-P_{3}$ hold, then the system of functions $V\left(L_{1, p}\right)$ is the Riesz basis in the space $H_{1}$.

Proof. Substituting functions (30) into the equations (8)-(13) makes sure that the numbers $\lambda_{k, m} \in \sigma$ are eigenvalues, if $k, m \in \mathbb{N}$.

In the space $H_{1}$ we define the operator $R\left(L_{1, p}\right):=E+S\left(L_{1, p}\right)$, which maps the system of functions $V\left(L_{0}\right)$ into $V\left(L_{1, p}\right)$.

The operator $R\left(L_{1, p}\right)$ has the form

$$
R\left(L_{1, p}\right):=\sum_{r, m} R\left(L_{1, p, m}\right) \times \pi_{2, r, m}
$$

where $\pi_{2, r, m}$ is the orthoprojector into the one-dimensional proper subspace in $H_{0}$, which corresponds to eigenfunction $\tau_{2, r, m}\left(x_{2}\right)$ of operator $B_{0}$.

We consider the operator $A_{p}: H_{1} \rightarrow H_{1}$, which has purely point spectrum $\sigma\left(A_{p}\right):=$ $\left\{\lambda_{k, m} \in \mathbb{R}: \lambda_{k, m}=\mu_{1, k}+\mu_{2, m}, k, m \in \mathbb{N}\right\}$ and the system of eigenfunctions

$$
V\left(A_{p}\right):=\left\{v_{s, r, k, m}\left(x_{1}, x_{2}, A_{p}\right):=v_{s, k}\left(x_{1}, A_{p, m}\right) \tau_{2, r, m}\left(x_{2}\right), s, r \in\{0,1\}, k, m \in \mathbb{N}\right\} .
$$

Let $R\left(A_{p}\right):=\sum_{r, m} R\left(A_{p, m}\right) \times \pi_{2, r, m}$. 
According to the Lemma 5, for an arbitrary $m \in \mathbb{N}$ the system of functions $W\left(L_{1, p, m}\right)$ exists, and it is biorthogonal to the system $V\left(L_{1, p, m}\right)$.

Therefore, we can define the elements of system $W\left(L_{1, p}\right)$, which is biorthogonal to system $V\left(L_{1, p}\right)$ in the space $H_{1}$ :

$$
w_{s, r, k, m}\left(x_{1}, x_{2}, L_{1, p}\right)=w_{s, k}\left(x_{1}, L_{1, p, m}\right) \tau_{2, r, m}\left(x_{2}\right), s, r \in\{0,1\}, k, m \in \mathbb{N} .
$$

Thus, the system $V\left(L_{1, p}\right)$ is complete and minimal in $H_{1}$.

Therefore, when the Assumptions $P_{2}$ and $P_{3}$ hold, then we obtain the inequality $\left|\eta_{p, k, m}\right| \leq$ $C_{9}<\infty$, for arbitrary $m, k \in \mathbb{N}$. Taking into account the estimates $\left\|R\left(A_{p}\right) ;\left[H_{1}\right]\right\|^{2} \leq C_{10}$, we obtain the statement: eigenfunctions (30) of operator $A_{p}$ are almost normalized, and system $V\left(A_{p}\right)$ is the Riesz basis of the space $H_{1}$.

We consider the operator $R\left(L_{1, p}\right)=E+S\left(L_{1, p}\right)=(E+Q)\left(E+S\left(A_{p}\right)\right)$. Then the operator $Q_{p}:=S\left(L_{1, p}\right)-S\left(A_{p}\right)$ is completely continuous, because the systems of functions $V\left(L_{1, p, m}\right)$, $V\left(A_{p, m}\right)$ are quadratically approximate and the operator $Q_{p, m}:=S\left(L_{1, p, m}\right)-S\left(A_{p, m}\right)$ is idempotent: $Q_{p, m}^{2}=0$.

According to the definition of function $v_{s, r, k, m}\left(x, L_{0}\right)$, we obtain

$$
\left\|Q_{p} v_{s, r, k, m}\left(x, L_{0}\right) ; H_{1}\right\|=O(m+k)^{-3}, m, k \rightarrow \infty .
$$

Then, for an arbitrary $h=\sum_{s, r, m, k} h_{s, r, k, m} v_{s, r, k, m}\left(x, L_{0}\right) \in H_{1}$, from Cauchy's inequality we can get the inequality

$$
\left\|Q_{p} h ; H_{1}\right\|^{2}=\left\|\sum_{s, r, k, m} h_{s, r, k, m} Q_{p} v_{s, r, k, m}\left(x, L_{0}\right) ; H_{1}\right\|^{2} \leq C_{11}\left\|h ; H_{1}\right\|^{2} .
$$

Thus $\left\|Q_{p} ;\left[H_{1}\right]\right\|^{2}<\infty,\left(L_{1, p}\right)=Q_{p}+R\left(A_{1, p}\right) \in\left[H_{1}\right], R\left(L_{1, p}\right)^{-1}=\left(E-S\left(A_{p}\right)(E-Q) \in\left[H_{1}\right]\right.$.

Proof. Proof of the Theorem 1. Let $R(L):=\prod_{p=1}^{n} R\left(L_{1, p}\right)$. The eigenfunctions of operator $L$ we can define in the form

$$
v_{s, r, k, m}(x, L):=R(L) v_{s, r, k, m}\left(x, L_{0}\right), r, s \in\{0,1\}, k, m \in \mathbb{N} \text {. }
$$

Taking into account, that operators $R\left(L_{1, p}\right)$ are elements of the group $\Gamma_{1, p}\left(L_{0}\right)$, we obtain

$$
R(L)=E+S(L), R^{-1}(L)=E-S(L), S(L):=\sum_{p=1}^{n} S\left(L_{1, p}\right) .
$$

Therefore, the system of eigenfunctions $V(L)$ is complete and minimal in $H_{1}$.

Proof. Proof of the Theorem 2. Let the Assumptions $P_{1}-P_{3}$ hold, then the system of eigenfunctions $V\left(L_{1, p}\right)$ is the Riesz basis in the space $H_{1}$, and $R(L)=\prod_{p=1}^{n} R\left(L_{1, p}\right) \in\left[H_{1}\right]$. Therefore, taking into account the theorem N.K. Bari [10], we obtain the statement of the theorem.

Let us define the elements of system $W(L)$, which is biorthogonal to system $V(L)$ in the space $H_{1}$ :

$$
w_{s, r, k, m}(x, L):=R(L) \tau_{1, s, k}\left(x_{1}\right) \tau_{2, r, m}\left(x_{2}\right), s, r \in\{0,1\}, \quad k, m \in \mathbb{N} .
$$


Remark 3. The positive numbers $C_{1}(L), C_{2}(L)$ exist, such that for function

$$
f(x)=\sum_{s, r, k, m} f_{s, r, k, m} v_{s, r, k, m}\left(x_{1}, x_{2}, L\right), f_{s, r, k, m}:=\left(f, w_{s, r, k, m}\left(x_{1}, x_{2}, L\right) ; H_{1}\right)
$$

the following inequality holds

$$
C_{2}(L)\left\|f ; H_{1}\right\|^{2} \leq \sum_{s, r, k, m}\left|f_{s, r, k, m}\right|^{2}, C_{3}(L)\left\|f ; H_{1}\right\|^{2} .
$$

Proof. Proof of the Theorem 3. We will use a solution of the problem (1)-(6) in the form of series

$$
u(x)=\sum_{s, r, k, m} u_{s, r, k, m} v_{s, r, k, m}\left(x_{1}, x_{2}, L\right) .
$$

If we substitute series (31), (32) into equation (1), we obtain

$$
u_{s, r, k, m}=\lambda_{k, m}^{-1} f_{s, r, k, m}
$$

Taking into account the Assumption $P_{3}$ and inequality $\lambda_{k, m}^{-1} \leq 1$, we can get

$$
\begin{gathered}
\left\|u ; H_{1}\right\|^{2} \leq C_{5}(L)\left\|f ; H_{1}\right\|^{2}, C_{5}(L)=C_{2}(L)^{-1}(L) C_{3}(L) C_{1}^{-2}(L), \\
\left\|D_{1}^{2 n} u ; H_{1}\right\|^{2} \leq C_{5}(L)\left\|f ; H_{1}\right\|^{2}, \\
\left\|D_{2}^{2 n} u ; H_{1}\right\|^{2} \leq C_{5}(L)\left\|f ; H_{1}\right\|^{2} .
\end{gathered}
$$

Therefore, $\left\|u ; H_{2}\right\|^{2} \leq 3 C_{5}(L)\left\|f ; H_{1}\right\|^{2}$.

\section{REFERENCES}

[1] Amanov D., Ashyralyev A. Well-posedness of boundary value problems for partial differential equations of even order. Electron. J. Diff. Equ. 2014, 108, 1-18.

[2] Amanov D.J., Yuldasheva A.V. Solvability and spectral properties of boundary value problems for equations of even order. Malays. J. Math. Sci. 2009, 3 (2), 227-248.

[3] Baranetskij Ya.O., Ivasiuk I.Ya., Kalenyuk P.I., Solomko A.V. The nonlocal boundary problem with perturbations of antiperiodicity conditions for the elliptic equation with constant coefficients. Carpathian Math. Publ. 2018, 10 (2), 215-234. doi:10.15330/cmp.10.2.215-234

[4] Baranetskij Ya.O., Kalenyuk P.I., Kopach M.I. The nonlocal boundary problem with multipoint perturbations for the differential equations with constant coefficients of even order. Mat. Metodi Fiz.-Mekh. Polya 2018, 61 (1), 16-39. (in Ukrainian)

[5] Baranetskij Ya.O., Kalenyuk P.I., Kolyasa L.I., Kopach M.I. The nonlocal problem for the differential-operator equation of the even order with the involution. Carpathian Math. Publ. 2017, 9 (2), 109-119. doi:10.15330/cmp.9.2.109119

[6] Baranetskij Ya.O., Demkiv I.I., Ivasiuk I.Ya., Kopach M.I. The nonlocal problem for the differential equations the order $2 n$ with an unbounded operator coefficients with the involution. Carpathian Math. Publ. 2018, 10 (1), 14-30. doi:10.15330/cmp.10.1.14-30

[7] Demkiv I.I., Ivasiuk I.Ya., Kopach M.I. Interpolation integral continued fraction with twofold node. MMC 2019, 6 (1), 1-13. doi:10.23939/mmc2019.01.001 
[8] Irgashev B.Yu. Boundary problem for equations of high-even order. Vestnik VGU. Seriya 1. Mathematica. Physica. 2016, 334 (3), 6-18. (in Russian) doi:10.15688/jvolsu1.2016.3.1

[9] Irgashev B.Yu. On spectral problem for one equation of high even order. Russian Math. (Iz. VUZ). 2016,60 (7), $37-46$.

[10] Gokhberg I.Ts., Krein M.G. Introduction to the Theory of Linear Non Self-Adjoint Operators. Nauka, Moscow, 1965. (in Russian)

[11] Koshanov B.D., Soldatov A.P. Boundary value problem with normal derivatives for a higher-order elliptic equation on the plane. Diff. Equations 2016, 52, (12), 1594-1609.

[12] Koshanov B., Soldatov A. About the generalized Dirichlet-Neumann problem for an elliptic equation of high order. AIP Conference Proceedings 1997, 020013 (2018). doi:10.1063/1.5049007

[13] Yuldasheva A.V. On solvability of the boundary value problem for one even-order equation. Vestnik KRAUNC. Fiz.-Mat. Nauki 2016, 12 (1), 41-47.

Received 03.04.2019

Баранецький Я.О., Каленюк П.І., Копач М.І., Соломко А.В. Нелокальна крайова задача зі збуреннями мішаних крайових умов для еліптичного рівняння зі сталими коефічієнтами. I // Карпатські матем. публ. - 2019. - Т.11, №2. - С. 228-239.

У роботі в одиничному квадраті $G$ методом Фур'є досліджується задача з нелокальними умовами, які є багатоточковими збуреннями мішаних крайових умов. Вивчено властивості узагальненого оператора перетворення $R: L_{2}(G) \rightarrow L_{2}(G)$, який відображає нормовані власні функції оператора $L_{0}$ задачі із мішаними крайовими умовами у власні функції оператора $L$ збуреної нелокальної задачі. Побудовано систему $V(L)$ власних функцій оператора $L$. Визначено умови, при яких система $V(L)$ повна та мінімальна в просторі $L_{2}(G)$, та умови, при яких вона є базисом Рісса у просторі $L_{2}(G)$. У випадку, якщо система $V(L) \in$ базисом Рісса в просторі $L_{2}(G)$, встановлено достатні мови, при яких нелокальна задача має єАиний розв' язок у вигляді ряду Фур'є за системою $V(L)$.

Ключові слова і фрази: диференщіальне рівняння з частинними похідними, кореневі функції, базис Рісса. 